\title{
Phaseolus vulgaris Recognizes Azorhizobium caulinodans Nod Factors with a Variety of Chemical Substituents
}

\author{
T. Laeremans, ${ }^{1}$ C. Snoeck, ${ }^{1}$ J. Mariën, ${ }^{1}$ C. Verreth, ${ }^{1}$ E. Martínez-Romero, ${ }^{2}$ J.-C. Promé,${ }^{3}$ and \\ J. Vanderleyden ${ }^{1}$ \\ ${ }^{1} \mathrm{~F}$. A. Janssens Laboratory of Genetics, Catholic University of Leuven, Kardinaal Mercierlaan 92, B-3001 \\ Heverlee, Belgium; ${ }^{2}$ Departamento de Genética Molecular, Centro de Investigación sobre Fijación de \\ Nitrógeno, Universidad Nacional Autónoma de México, Ap. 565-A Cuernavaca, Morelos, Mexico; \\ ${ }^{3}$ Laboratoire des Interactions Moléculaires et Réactivité Chimique et Photochimique, UMR 5623, \\ Université Paul Sabatier, 118 Route de Narbonne, 31062 Toulouse Cedex, France \\ Accepted 3 May 1999.
}

\begin{abstract}
Phaseolus vulgaris is a promiscuous host plant that can be nodulated by many different rhizobia representing a wide spectrum of Nod factors. In this study, we introduced the Rhizobium tropici CFN299 Nod factor sulfation genes nodHPQ into Azorhizobium caulinodans. The A. caulinodans transconjugants produce Nod factors that are mostly if not all sulfated and often with an arabinosyl residue as the reducing end glycosylation. Using $A$. caulinodans mutant strains, affected in reducing end decorations, and their respective transconjugants in a bean nodulation assay, we demonstrated that bean nodule induction efficiency, in decreasing order, is modulated by the Nod factor reducing end decorations fucose, arabinose or sulfate, and hydrogen.
\end{abstract}

Additional keywords: nodH, nodPQ.

During the early stages of the Rhizobium spp.-legume symbiosis, newly formed plant organs, the nodules, emerge after a reciprocal signal exchange. Via the infection thread, the bacteria can invade the nodules, differentiate into bacteroids, and subsequently start to fix nitrogen (reviewed by Mylona et al. 1995). Truchet et al. (1991) demonstrated that the Nod factors (NFs) are the essential bacterial signaling molecules for nodule induction. NFs are chitin molecules, consisting of a backbone of up to six $\beta$ - $(1,4)$-linked $N$-acetyl glucosamine sugar units, coupled to a fatty acyl chain at the nonreducing end and with strain-specific substitutions at both the nonreducing and reducing $N$-acetyl glucosamine (GlcNAc) residue (Lerouge et al. 1990; Spaink et al. 1991). Recently, two novel NF structures were discovered in Mesorhizobium loti. One NF structure consisted of only two GlcNAc sugar units while the other was an NF carrying a fucosyl group at a nonterminal GlcNAc (Olsthoorn et al. 1998). The enzymes necessary for the production of the NF backbone are encoded by the nodABC genes

Corresponding author: J. Vanderleyden: Telephone: (32) 16321631 ; Fax: (32) 163219 66; E-mail: jozef.vanderleyden@agr.kuleuven.ac.be found in all nodulating rhizobial strains. These genes are referred to as "common" nod genes (Kondorosi 1991) although recently Ritsema and co-workers (1996) found that the replacement of Rhizobium leguminosarum bv. viciae nodA by its Bradyrhizobium japonicum homologue resulted in the loss of Vicia spp. nodulation. Additionally, Debellé et al. (1996) proved that the NodA protein of Sinorhizobium meliloti contributes to host range by determining the oligomerization of the NF backbone. The host-specific nodulation genes are responsible for the side groups encountered on the GlcNAc residues of the NF core molecule (for recent reviews, see Dénarié et al. 1996 and Mergaert et al. 1997b).

Phaseolus vulgaris is a promiscuous host plant that can be nodulated $\left(\mathrm{Nod}^{+} \mathrm{Fix}^{+}\right.$or $\left.\mathrm{Nod}^{+} \mathrm{Fix}^{-}\right)$by a wide spectrum of rhizobia (Martínez et al. 1985; Michiels et al. 1998; reviewed by Laeremans and Vanderleyden 1998). The Sesbania rostrata isolate A. caulinodans ORS571 (Dreyfus et al. 1988) is able to elicit Fix ${ }^{-}$nodules on bean plants (Waelkens et al. 1995; Michiels et al. 1998). A. caulinodans ORS571 produces a complex mixture of tetra- and pentameric NFs, acylated with stearic, vaccenic, or palmitic acid, methylated and carbamoylated at the nonreducing end and often with a fucosyl and/or arabinosyl moiety coupled to the reducing end. The A. caulinodans NF can be arabinosylated at its reducing end on $\mathrm{C} 3$ and possibly on C6. However, when fucose is present, arabinose is always linked to the C3 (Mergaert et al. 1997a). The A. caulinodans ORS571-1.11Z nodZ mutant strain is unable to couple an arabinose group to the reducing end and fucosylation is severely reduced (Mergaert et al. 1996). The ORS571-4.2K nolK mutant does not produce fucosylated NFs, but arabinosylation remains unaffected (Mergaert et al. 1996). The NFs of the A. caulinodans ORS571-1.11Z-4.2K double nodZnolK mutant are free of reducing end substitutions (W. D'Haeze and M. Holsters, personal communication).

$R$. tropici CFN299 produces a mixture of sulfated and nonsulfated NFs (Poupot et al. 1993). We isolated the $R$. tropici nodHPQ genes and demonstrated that they are required for NF sulfation. Plant phenotypic analysis showed that the NF 
sulfate decoration is not essential for bean nodulation but modulates, in a cultivar-dependent manner, nodule formation efficiency on bean roots (Laeremans et al. 1996).

In this study, we transferred the $R$. tropici genetic locus for NF sulfation to $A$. caulinodans $\left(\mathrm{Nod}^{+} \mathrm{Fix}^{-}\right.$on bean) wild-type and nod mutant strains in order to determine the effect of different NF reducing end substitutions for nodulation of bean. We compared NF structure and bean nodulation phenotype of the $A$. caulinodans acceptor and transconjugant strains.

Two primers, HPQ1 (5'-GGAATTCAGATGTATCCTGT ACCACCGCGCG-3') and HPQ2 (5'-CCTAAGCTTGATT TTCGGCCCTCCCCTGTTGAC-3') were used on pTL3 (Laeremans et al. 1996) as the template for a polymerase chain reaction (PCR) experiment. Recognition sites for restriction enzymes are indicated in bold. We performed the PCRs with the Expand High Fidelity PCR system (Boehringer, Mannheim, Germany) according to the manufacturer's protocol to increase fidelity of DNA synthesis. DNA was synthesized during 34 cycles of a 1-min denaturation step at $94^{\circ} \mathrm{C}$, a 1-min primer annealing step at $55^{\circ} \mathrm{C}$, followed by 4 min of primer extension at $72^{\circ} \mathrm{C}$. An additional $5 \mathrm{~min}$ at $72^{\circ} \mathrm{C}$ was performed to complete partial extension products. An expected PCR fragment of approximately $4 \mathrm{~kb}(4,037 \mathrm{bp})$, containing the $R$. tropici CFN299 nodHPQ genes with 400 upstream nucleotides, was purified, double-digested with EcoRI-HindIII, and cloned into pUC19. The resulting construct was transformed to E. coli that was grown at $37^{\circ} \mathrm{C}$ on Luria-Bertani medium (Sambrook et al. 1989) with tetracycline (Tc; $10 \mathrm{mg} \mathrm{l}^{-1}$ ). From both insert extremes, the nucleotide sequence was determined with the universal or reverse primer on an ALF automated sequencer (Pharmacia Biotech, Uppsala, Sweden). The obtained sequences were identical to the corresponding regions of the submitted $R$. tropici nodHPQ GenBank sequence (U47272). We then recloned the 4-kb EcoRI-HindIII fragment into pLAFR3 (Staskawicz et al. 1987), resulting in pRTHPQ. Subsequently, pRTHPQ was introduced by triparental mating into A. caulinodans ORS571, ORS571-1.11Z, ORS571-4.2K, and ORS5711.11Z-4.2K as described by van Rhijn et al. (1993). A. caulinodans strains were grown on TY (Beringer 1974) containing ampicillin $\left(100 \mathrm{mg} \mathrm{l}^{-1}\right)$. We added kanamycin $\left(50 \mathrm{mg} \mathrm{l}^{-1}\right)$ for the mutant strains and $\mathrm{Tc}\left(10 \mathrm{mg} \mathrm{l}^{-1}\right)$ for the transconjugants. The introduced plasmid was isolated from A. caulinodans $\left(\mathrm{Tc}^{\mathrm{r}}\right)$ transconjugants by an alkaline lysis procedure (Sambrook et al. 1989) and subsequently verified by restriction analysis.

Isolation and separation of the flavonoid induced ${ }^{14} \mathrm{C}$ acetate-labeled Nod metabolites of the A. caulinodans transconjugants by reverse-phase thin-layer chromatography (RPTLC) demonstrated that their NFs became more hydrophilic, compared with those of the corresponding recipient strains. Labeling with ${ }^{35} \mathrm{~S}$-sulfate suggested that the presence of pRTHPQ was responsible for sulfation of most if not all of the NF backbones of the transconjugants of ORS571-1.11Z, ORS5714.2K, and ORS571-1.11Z-4.2K (Fig. 1). For ORS571(pRTHPQ), limited amounts of naringenin-induced metabolites are not labeled with ${ }^{35}$ S-sulfate (Fig. 1, spot NS1).

By reverse-phase high-pressure liquid chromatography (RPHPLC), the NF fractions PI and PII of ORS571 elute around min 25 and 27, respectively. PI is a mixture of C18:1 and C16:0 acylated NFs with the reducing end glycosylated (fucosylated, arabinosylated, or both) or unsubstituted. PII is a mixture of C18:0 acylated NFs with the reducing end glyco- sylated (fucosylated, arabinosylated, or both) or unsubstituted (Mergaert et al. 1997a). Under identical conditions, 12 fractions from ORS571(pRTHPQ), eluting between min 15 and 30, were collected. As suggested by the NF TLC profile (Fig. 1), sulfated NFs are more hydrophilic and should elute earlier than the nonsulfated NFs of ORS571. Indeed, liquid secondary ion mass spectrometry (LSIMS) in the positive ionization mode of the 12 collected fractions indicated that, under the conditions assayed, Nod metabolite-containing fractions elute between min 18 and 22 (data not shown). The fragmentation pattern of the $[\mathrm{M}+\mathrm{H}]^{+}$ions, showing successive losses of 203 mass units (characteristic for a GlcNAc oligomeric backbone), all ended at $m / z$ 483, 485 (mainly), or 457. These masses correspond to a carbamoylated and methylated GlcNAc extremity of the NFs acylated with C18:1, C18:0, or C16:0 fatty acids, respectively (data not shown). These $m / z$ values indicate that the nonreducing end decorations of the NFs of ORS571(pRTHPQ) are identical to those of the ORS571 NFs. Negative ion LSIMS was performed on the RP-HPLC fractions eluting between min 18 and 22 to verify whether the spectra of these fractions show molecular masses corresponding to those of decorated lipo-oligosaccharides. Deduced from positive and negative ion mass spectrometry, the most abundantly detected NFs of ORS571(pRTHPQ) have molecular masses of 1,392,1,394, 1,524, and 1,526 Da. The molecules corresponding to a molecular mass of 1,392 Da (spot S1 in Figure 1) and 1,394 Da (spot S3 in Figure 1) represent NFs NodARc-V(C18:1;Me;Carb;S) and NodARc$\mathrm{V}(\mathrm{C} 18: 0 ; \mathrm{Me} ; \mathrm{Carb} ; \mathrm{S})$, respectively. The two molecules with a shift of 132 mass units, 1524 (S2) and 1526 (S4), are the arabinosylated species, NodARc-V(C18:1;Me;Carb;Ara;S) and NodARcV(C18:0;Me;Carb;Ara;S). Thus, all detected NFs were pentameric with a sulfate and occasionally an arabinose moiety as reducing end decorations. The nonreducing end was C18:0 or C18:1 acylated, with a carbamoyl and an $N$-methyl group additionally linked. No $[\mathrm{M}+\mathrm{H}]^{+}$or $[\mathrm{M}-\mathrm{H}]^{-}$ions with $\mathrm{m} / \mathrm{z}$ values corresponding to nonsulfated or fucosylated NFs, produced by the wild-type ORS571, were detected. [M $\mathrm{H}]^{-}$ions at $\mathrm{m} / \mathrm{z} 1365,1188$, and 1190 , corresponding respectively to NodARc-V(C16:0;Me;Carb;S), NodARcIV(C18:1;Me;Carb;S), and NodARc-IV(C18:0;Me;Carb;S), were also detected but their quantities were estimated below the $10 \%$ level. The additionally arabinosylated analogues of these NFs, if present, are not distinguishable from the background of the spectra. After repeated purification of the butanol extracts containing the NFs of ORS571(pRTHPQ), we consistently detected limited amounts of nonsulfated, naringenin-induced metabolites on TLC plates (Fig. 1). By LSIMS, however, we could not detect nonsulfated NFs. Whether these spots correspond to nonsulfated NFs not distinguishable from the background of the spectra or to other metabolites is not known. To locate the sulfate group on the arabinosylated species NodARc-V(C18:0;Me;Carb;S;Ara), its $[\mathrm{M}+\mathrm{H}]^{+}$ion at $\mathrm{m} / \mathrm{z} 1527$ was selected and fragmented by collision (Fig. 2). The base peak was the loss of $\mathrm{SO}_{3}$ (80 mass units). Ion $\mathrm{B} 4$ at $\mathrm{m} / \mathrm{z}, 1094$ corresponded to the loss of the reducing $\mathrm{N}$-acetylglucosaminyl residue bearing both the arabinosyl and sulfate moieties. The diagnostic peak for locating the sulfate group was at $m / z$ 1395. It was due to the loss of the arabinose residue (132 mass units), indicating that a sulfate group was not coupled to the arabinose. The loss of arabinose plus $\mathrm{SO}_{3}(\mathrm{~m} / z, 1315)$ may 
be interpreted as due to two consecutive eliminations and is not of diagnostic value. The ion at $m / z, 1226$ was more puzzling: it is due to the expulsion of the reducing sulfated GlcNAc while keeping the arabinosyl group attached to the rest of the molecule. In fact, a similar behavior was found for a fucosyl residue of an NF (Bec-Ferté et al. 1994; Cárdenas et al. 1995) and explained by a gas phase isomerization of the molecule before fragmentation, leading to migration of fucose on the chito backbone. These data suggest that the sulfate and arabinosyl moieties are located on the NF reducing end and that both decorations are directly linked to the GlcNAc. We cannot exclude, however, that NFs with sulfate coupled on arabinose are also present in minor quantities.

In vitro assays with the $A$. caulinodans or $B$. japonicum NodZ protein indicate that fucosylation of the NF occurs after oligomerization of the GlcNAc subunits (Mergaert et al. 1996; López-Lara et al. 1996; Quesada-Vincens et al. 1997; Quinto et al. 1997). Ehrhardt et al. (1995) and Schultze et al. (1995) showed that NodH can sulfate both lipo-chitooligosaccharidic and chitooligosaccharidic molecules in vitro. Our results suggest that, in ORS571(pRTHPQ), the sulfate residue occupies NF reducing end C6 positions before fucosylation can occur. Hence, the introduced sulfotransferase gene nodH completely blocks fucosylation by the NF fucosyltransferase in ORS571. Nevertheless, we cannot exclude that the introduction of the $R$. tropici CFN299 genes that encode for sulfate activation enzymes (nodPQ) in A. caulinodans ORS571 could be responsible for a dramatic increase in the ratio of substrates for NodH to NodZ transferases, resulting in the absence of fucosylated NFs. Based on the NF TLC profiles, the presence of
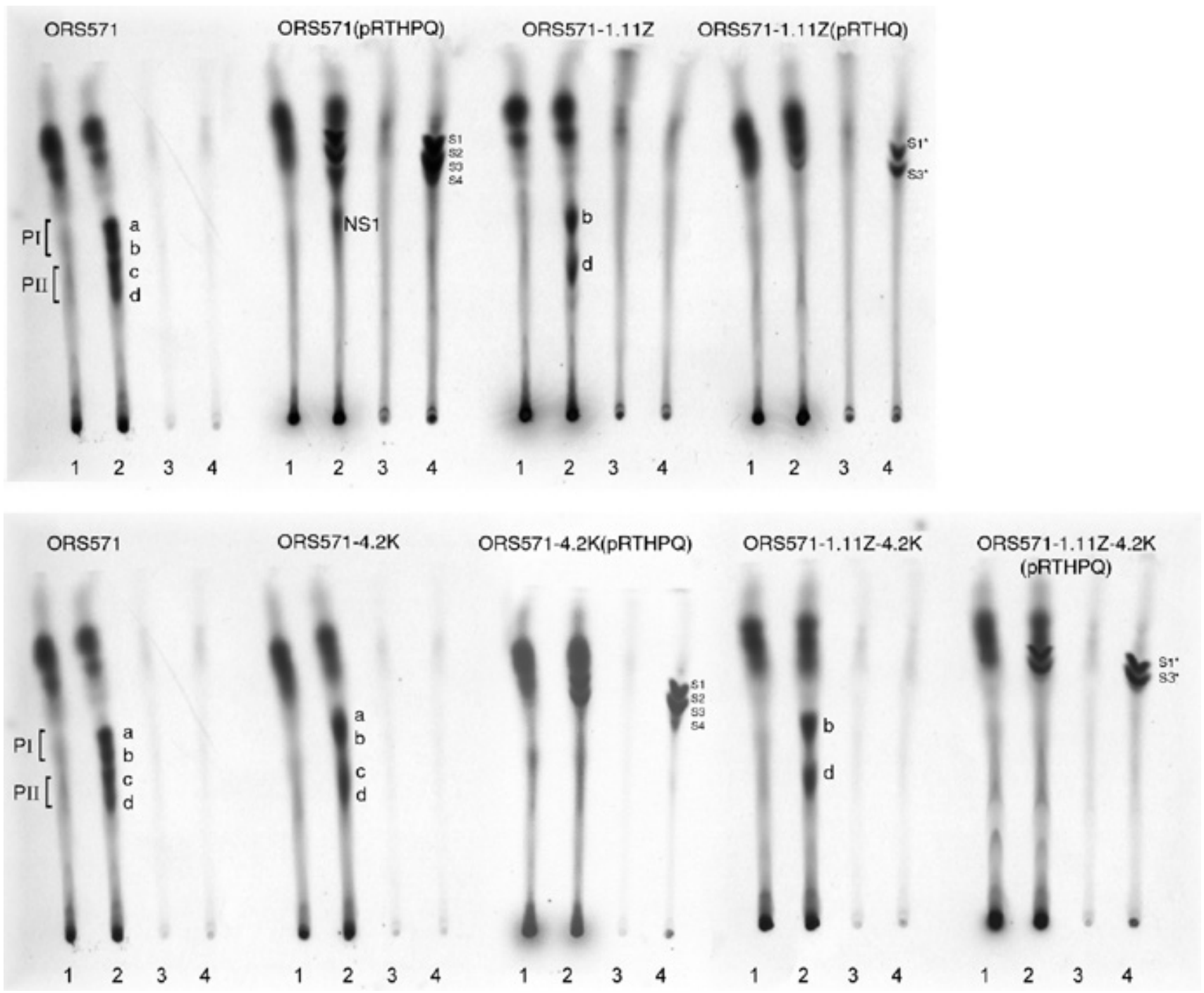

Fig. 1. Reverse-phase thin-layer chromatography patterns of Azorhizobium caulinodans wild-type, mutant, and transconjugant strains. Butanol extracts of naringenin-induced (final concentration $10 \mu \mathrm{M}$ ), radioactively labeled cell cultures were purified and separated as described by Mergaert et al. (1993). After overnight induction and labeling, no significant differences in number of cells were measured. Radioactively labeled compounds were visualized with Hyperfilm (Amersham, UK) after $96 \mathrm{~h}$ of exposure. ${ }^{14} \mathrm{C}$-acetate-labeled: lanes 1 (noninduced) and 2 (induced); ${ }^{35} \mathrm{~S}$-sulfate-labeled: lanes 3 (noninduced) and 4 (induced). PI and PII refer to the A. caulinodans Nod factor (NF) mixtures as reported by Mergaert et al. (1997a). PI is a mixture of C18:1 and C16:0 acylated NFs with the reducing end (a) glycosylated (fucosylated or arabinosylated or both) or (b) unsubstituted. PII is a mixture of C18:0 acylated NFs with the reducing end (c) glycosylated (fucosylated or arabinosylated or both) or (d) unsubstituted. Spots marked with S1 to S4 correspond to sulfated NFs the structures of which are described in the text. Carbamoyl and methyl substitutions are identical in a, b, c, d, and S1 to S4. For spots indicated with an asterisk, structures were not determined by liquid secondary ion mass spectrometry but can be deduced from structures of the NFs of the A. caulinodans acceptor strains. 
glycosyl transferase activity with NF as a substrate does not prevent sulfation, but a competition for substrate exists between sulfo- and glycosyltransferases. Indeed, chemical characterization of the NFs of ORS571(pRTHPQ) by LSIMS showed that the NFs detected are sulfated and additionally can bear an arabinosyl residue on the reducing end, while the wild-type A. caulinodans ORS571 produces unsubstituted, fucosylated and/or arabinosylated NFs.

To determine the quantitative effect of the different NF reducing end decorations on bean nodulation, we inoculated bean seedlings monoxenically with $A$. caulinodans wild-type, mutant, and transconjugant strains harboring pRTHPQ, differently affected in NF reducing end decorations (Fig. 3). ORS571-1.11Z and ORS571-1.11Z-4.2K, producing NFs that are either residually fucosylated or without any NF glycosylation, induced only a very low number of white, $\mathrm{Fix}^{-}$nodules on bean roots. An intermediate number of nodules were formed after inoculation with ORS571-4.2K, producing NFs with arabinosyl as the reducing end glycosylation. The inoculation of bean plants with ORS571(pRTHPQ) resulted in a significant decrease in nodule number compared with the acceptor strain. ORS571(pRTHPQ) and ORS5714.2K(pRTHPQ) induced statistically indistinguishable numbers of nodules on bean, but a higher number than induced by ORS571-4.2K, which produces NFs with arabinose as the only reducing end decoration. LSIMS spectra of NFs purified from ORS571-4.2K(pRTHPQ) indicate that the structure of the NFs is identical to that of NFs produced by ORS571 carrying pRTHPQ (data not shown). For ORS571-1.11Z(pRTHPQ), the number of nodules formed was not significantly different from that of the nodules formed by ORS571-1.11Z. Similar results were obtained in a concurrent study by Fernández-López et al. (1998). The authors found that fucosylated A. caulinodans NFs were more efficient than arabinosylated NFs in their ability to induce nodules on bean plants. The significant

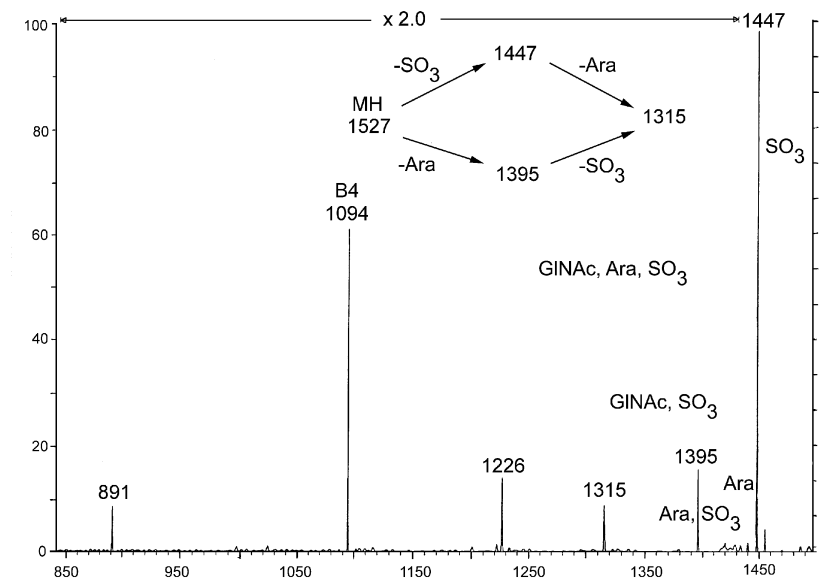

Fig. 2. MS/MS (mass spectra/mass spectrometry) spectrum of the Nod metabolite corresponding to the $[\mathrm{M}+\mathrm{H}]^{+}$ion at $\mathrm{m} / \mathrm{z}$ 1525. Nod factors (NFs) were isolated from naringenin-induced 10-liter cell cultures, as described by Mergaert et al. (1997a). MS were measured on an Autospec instrument (Micromass, Manchester, UK), fitted with a liquid secondary ion mass spectrometry ion source and a cesium gun working at $25 \mathrm{kV}$. Acceleration voltage was $8 \mathrm{kV}$. A mixture of metanitrobenzyl alcohol and glycerol was used as the matrix. In positive ion mode, the matrix was acidified with $10 \%$ trichloroacetic acid. Addition of acid was omitted for negative ion measurements. Ionic decompostion studies by collision on helium gas were performed at constant $\mathrm{B} / \mathrm{E}$ scanning. difference in bean nodule number between ORS5711.11Z(pRTHPQ) and ORS571-1.11Z-4.2K(pRTHPQ) (kindly provided by W. D'Haeze and M. Holsters), which putatively produce identical NFs, could be due to different amounts of NF production. Indeed, although in all cases NFs were prepared from cell cultures containing an equal amount of cells, the intensity of the spots in TLC indicates that the amount of NFs purified from ORS571-1.11Z-4.2K(pRTHPQ) NFs is higher than from ORS571-1.11Z(pRTHPQ) (Fig. 1). Similar effects were previously shown by Relić et al. (1994). Although not statistically different with the method we used, a higher number of nodules were consistently induced by ORS571-1.11Z(pRTHPQ) compared with ORS571-1.11Z. No differences between bean cultivars N-8-116 and Negro Jamapa were observed after the inoculation with the wild-type, mutant, and transconjugant strains (data not shown). In conclusion, A. caulinodans transconjugant strains producing sulfated NFs always induced less nodules than strains producing sulfated NFs that are additionally glycosylated at their reducing end. The NF reducing end GlcNAc substitutions responsible for the distinct nodulation efficiencies, in decreasing order, are as follows: fucosyl, arabinosyl or sulfate residue, and $\mathrm{H}$.

The sulfate residue on the $S$. meliloti NF backbone protects the NF against breakdown by plant root chitinases (Staehelin et al. 1994). Whether the sulfate residue or any C6 decoration specifically protects NFs against bean root chitinase breakdown remains to be elucidated.

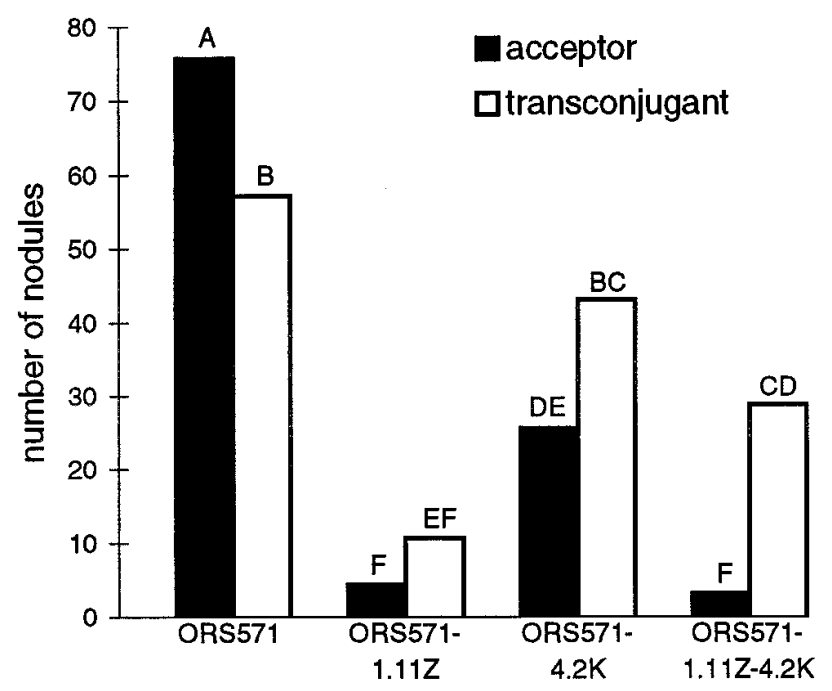

Fig. 3. Number of nodules formed on Phaseolus vulgaris cv. N-8-116 inoculated with Azorhizobium caulinodans wild-type, mutant, and transconjugant strains, harboring pRTHPQ. Seeds of $P$. vulgaris cvs. N-8-116 and Negro Jamapa were surface sterilized, germinated, and subsequently axenically transferred to cotton-Fahreus as previously described by Laeremans et al. (1997). Bean roots were monoxenically inoculated with $100 \mu \mathrm{l}$ of the appropriate cell suspension $\left(\mathrm{OD}_{600}=0.05\right)$. Beans were grown under a day/night regime of $12 / 12 \mathrm{~h}$ at $28^{\circ} \mathrm{C}$ and $75 \%$ relative humidity. Number of nodules was determined 21 days post inoculation. Noninoculated bean plants did not form nodules. Statistical data analysis was performed by analysis of variance with the general linear model procedure (SAS institute, Cary, NC). Statistical significance of the differences between inoculation treatments was determined with Duncan's test $(P<0.05)$. Each bar represents the average nodule number of 10 bean plants inoculated monoxenically. Each experiment was performed three times. One experiment is represented. Treatments with the same letter above the bars are not significantly different $(P<0.05)$. 


\section{ACKNOWLEDGMENTS}

This work was partially financed by a VLIR-ABOS grant from the Belgium Government. We thank C. Verplancken for help with Nod factor purification, P. Mergaert for supplying the A. caulinodans mutants, and M. Holsters and W. D'Haeze for helpful discussions (all affiliated with the Laboratory of Genetics, Universiteit Gent, Belgium). We acknowledge Ellen Luyten and Manuel Fernández-López for helpful discussions and Jos Desair and Yves Geunes for help with the figures.

\section{LITERATURE CITED}

Bec-Ferté, M.-P., Krishnan, H. B., Promé, D., Savagnac, A., Pueppke, S G., and Promé, J.-C. 1994. Structures of nodulation factors from the nitrogen-fixing soybean symbiont Rhizobium fredii USDA257. Biochemistry 33:11782-11788.

Beringer, J. E. 1974. R factor transfer in Rhizobium leguminosarum. J. Gen. Microbiol. 84:188-198.

Cárdenas, L., Domínguez, J., Quinto, C., López-Lara, I. M., Lugtenberg, B. J. J., Spaink, H. P., Rademaker, G. J., Haverkamp, J., and ThomasOates, J. E. 1995. Isolation, chemical structures and biological activity of the lipo-chitin oligosaccharide nodulation signals from Rhizobium etli. Plant Mol. Biol. 29:453-464.

Debellé, F., Plazanet, C., Roche, P., Pujol, C., Savagnac, A., Rosenberg, C., Promé, J.-C., and Dénarié, J. 1996. The NodA proteins of Rhizobium meliloti and Rhizobium tropici specify the N-acylation of Nod factors by different fatty acids. Mol. Microbiol. 22:303-314.

Dénarié, J., Debellé, F., and Promé, J.-C. 1996. Rhizobium lipo-chitooligosaccharide nodulation factors: Signaling molecules mediating recognition and morphogenesis. Annu. Rev. Biochem. 65:503-535.

Dreyfus, B., Garcia, J. L., and Gillis, M. 1988. Characterization of Azorhizobium caulinodans gen. nov., sp. nov., a stem-nodulating nitrogen-fixing bacterium isolated from Sesbania rostrata. Int. J. Syst. Bacteriol. 38:89-98

Ehrhardt, D. W., Atkinson, E. M., Faull, K. F., Freedberg, D. I., Sutherlin, D. P., Armstrong, R., and Long, S. R. 1995. In vitro sulfotransferase activity of NodH, a nodulation protein of Rhizobium meliloti required for host-specific nodulation. J. Bacteriol. 177:6237-6245.

Fernández-López, M., D’Haeze, W., Van Montagu, M., and Holsters, M. 1998. Changes in the glycosylation pattern at the reducing end of azorhizobial Nod factors affect nodulation efficiency. FEMS Microbiol. Lett. 158:237-242.

Kondorosi, A. 1991. Regulation of nodulation genes in rhizobia. Pages 325-340 in: Molecular Signals in Plant-Microbe Communication. D. P. S. Verma, ed. CRC Press, Boca Raton, FL.

Laeremans, T., Caluwaerts, I., Verreth, C., Rogel, M. A., Vanderleyden, J., and Martínez-Romero, E. 1996. Isolation and characterization of the Rhizobium tropici Nod factor sulfation genes. Mol. Plant-Microbe Interact. 9:492-500.

Laeremans, T., Coolsaet, N., Verreth, C., Snoeck, C., Hellings, N., Vanderleyden, J., and Martínez-Romero, E. 1997. Functional redundancy of genes for sulfate activation enzymes in Rhizobium sp. BR816. Microbiology 143:3933-3942.

Laeremans, T., and Vanderleyden, J. 1998. Infection and nodulation signalling in Rhizobium-Phaseolus vulgaris symbiosis. World J. Microbiol. Biotechnol. 14:787-808.

Lerouge, P., Roche, P., Faucher, C., Maillet, F., Truchet, G., Promé, J.-C., and Dénarié, J. 1990. Symbiotic host-specificity of Rhizobium meliloti is determined by a sulphated and acylated glucosamine oligosaccharide signal. Nature 344:781-784.

López-Lara, I. M., Blok-Tip, L., Quinto, C., Garcia, M. L., Stacey, G., Bloemberg, G. V., Lamers, G. E. M., Lugtenberg, B. J. J., ThomasOates, J. E., and Spaink, H. P. 1996. NodZ of Bradyrhizobium extends the nodulation host range of Rhizobium by adding a fucosyl residue to nodulation signals. Mol. Microbiol. 21:397-408.

Martínez, E., Pardo, M. A., Palacios, R., and Cevallos, M. A. 1985. Reiteration of nitrogen fixation gene sequences and specificity of Rhizobium in nodulation and nitrogen fixation in Phaseolus vulgaris. J. Gen. Microbiol. 131:1779-1786.

Mergaert, P., D’Haeze, W., Fernández-López, M., Geelen, D., Goethals, K., Promé, J.-C., Van Montagu, M., and Holsters, M. 1996. Fucosyla- tion and arabinosylation of Nod factors in Azorhizobium caulinodans: Involvement of nolK, nodZ as well as noe $C$ and/or downstream genes. Mol. Microbiol. 21:409-419.

Mergaert, P., Ferro, M., D'Haeze, W., Van Montagu, M., Holsters, M., and Promé, J.-C. 1997a. Nod factors of Azorhizobium caulinodans ORS571 can be glycosylated with an arabinosyl group, a fucosyl group, or both. Mol. Plant-Microbe Interact. 10:683-687.

Mergaert, P., Van Montagu, M., and Holsters, M. 1997b. Molecular mechanisms of Nod factor diversity. Mol. Microbiol. 25:811-817.

Mergaert, P., Van Montagu, M., Promé, J.-C., and Holsters, M. 1993. Three unusual modifications, a D-arabinosyl, an $\mathrm{N}$-methyl, and a carbamoyl group, are present on the Nod factors of Azorhizobium caulinodans strain ORS571. Proc. Natl. Acad. Sci. USA 90:1551-1555.

Michiels, J., Dombrecht, B., Vermeiren, N., Xi, C., Luyten, E., and Vanderleyden, J. 1998. Phaseolus vulgaris is a non-selective host for nodulation. FEMS Microbiol. Ecol. 26:193-205.

Mylona, P., Pawlowski, K., and Bisseling, T. 1995. Symbiotic nitrogen fixation. Plant Cell 7:869-885

Olsthoorn, M. M. A., López-Lara, I. M., Petersen, B. O., Bock, K., Haverkamp, J., Spaink, H. P., and Thomas-Oats, J. E. 1998. Nove branched Nod factor structure results from $\alpha-(1 \rightarrow 3)$ fucosyl transferase activity: The major lipo-chitin oligosaccharides from Mesorhizobium loti strain NZP2213 bear an $\alpha-(1 \rightarrow 3)$ fucosyl substituent on a nonterminal backbone residue. Biochemistry 37:9024-9032.

Poupot, R., Martínez-Romero, E., and Promé, J.-C. 1993. Nodulation factors from Rhizobium tropici are sulfated or nonsulfated chitopentasaccharides containing an $N$-methyl- $N$-acylglucosaminyl terminus. Biochemistry 32 : 10430-10435.

Quesada-Vincens, D., Fellay, R., Nasim, T., Viprey, V., Burger, U., Promé, J.-C., Broughton, W. J., and Jabbouri, S. 1997. Rhizobium sp. strain NGR234 NodZ protein is a fucosyltransferase. J. Bacteriol. 179:5087-5093.

Quinto, C., Wijfjes, A. H. M., Bloemberg, G. V., Blok-Tip, L., LópezLara, I. M., Lugtenberg, B. J. J., Thomas-Oates, J. E., and Spaink, H. P. 1997. Bacterial nodulation protein NodZ is a chitin oligosaccharide fucosyltransferase which can also recognize related substrates of animal origin. Proc. Natl. Acad. Sci. USA 94:4336-4341.

Relić, B., Staehelin, C., Fellay, R., Jabbouri, S., Boller, T., and Broughton, W. J. 1994. Pages 69-75 in: Proc. 1st Eur. Nitrogen Fixation Conf.. G. B. Kiss and G. Endre, eds. Officina Press, Szeged, Hungary.

Ritsema, T., Wijfjes, A. H. M., Lugtenberg, B. J. J., and Spaink, H. P. 1996. Rhizobium nodulation protein NodA is a host-specific determinant of the transfer of fatty acids in Nod factor biosynthesis. Mol. Gen. Genet. 251:44-51.

Sambrook, J., Fritsch, E. F., and Maniatis, T. A. 1989. Molecular Cloning: A Laboratory Manual. 2nd ed. Cold Spring Harbor Laboratory, Cold Spring Harbor, NY.

Schultze, M., Staehelin, C., Röhrig, H., John, M., Schmidt, J., Kondorosi, E., Schell, J., and Kondorosi A. 1995. In vitro sulfotransferase activity of Rhizobium meliloti NodH protein: Lipochitooligosaccharide nodulation signals are sulfated after synthesis of the core structure. Proc. Natl. Acad. Sci. USA 92:2706-2709.

Spaink, H. P., Sheeley, D. M., van Brussel, A. A. N., Glushka, J., York, W. S., Tak, T., Geiger, O., Kennedy, E. P., Reinhold, V. N., and Lugtenberg, B. J. J. 1991. A novel highly unsaturated fatty acid moiety of lipo-oligosaccharide signals determines host specificity of Rhizobium. Nature 354:125-130.

Staehelin, C., Schultze, M., Kondorosi, E., Mellor, R. B., Boller, T., and Kondorosi, A. 1994. Structural modifications in Rhizobium meliloti Nod factors influence their stability against hydrolysis by root chitinases. Plant J. 5:319-330.

Staskawicz, B., Dahlbeck, D., Keen, N., and Napoli, C. 1987. Molecular characterization of cloned avirulence genes from race 0 and race 1 of Pseudomonas syringae pv. glycinea. J. Bacteriol. 169:5789-5794.

Truchet, G., Roche, P., Lerouge, P., Vasse, J., Camut, S., de Billy, F. Promé, J.-C., and Dénarié, J. 1991. Sulphated lipo-oligosaccharide signals of Rhizobium meliloti elicit root nodule organogenesis in alfalfa. Nature 351:670-673.

van Rhijn, P. J. S., Feys, B., Verreth, C., and Vanderleyden, J. 1993. Multiple copies of nodD in Rhizobium tropici CIAT899 and BR816. J. Bacteriol. 175:438-447.

Waelkens, F., Voets, T., Vlassak, K., Vanderleyden, J., and van Rhijn, P. 1995. The nodS gene of Rhizobium tropici strain CIAT899 is necessary for nodulation on Phaseolus vulgaris and on Leucaena leucocephala. Mol. Plant-Microbe Interact. 8:147-154. 